\title{
Relationship between sweat function determined by the sudoscan and parameters of the metabolic syndrome in people with type 2 diabetes
}

\author{
Ovono Abessolo Félix ${ }^{1, *}$, Mbang Bengone Aude Syntia ${ }^{2}$, Ndong Atome Guy Roger ${ }^{3}$, Batou Alvine Sybille ${ }^{4}$, \\ Ngou Milama Edouard ${ }^{5}$
}

\author{
1,3 Doctor, ${ }^{2,4}$ Miss, ${ }^{5}$ Professor, ${ }^{1,2,4,5}$ Dept. of Chemistry-Biochemistry, Faculty of Medicine, University of Health Sciences, \\ Libreville, Gabon, ${ }^{3}$ Laboratory of Research in Biochemistry (LAREBIO), University of Sciences and Technology of Masuku, \\ Franceville, Gabon
}

\section{*Corresponding Author: Ovono Abessolo Félix}

Email: ovonab@yahoo.fr

Received: $16^{\text {th }}$ February, 2018

Accepted: $24^{\text {th }}$ May, 2018

\begin{abstract}
Objective: The aim of this study was to investigate the relationship between the sweat function and parameters of the metabolic syndrome in type 2 diabetes patients.

Materials and Methods: This was a cross-sectional study, which took place from July 2016 to December 2016. It was carried out on type 2 diabetes patients who were under treatment. The sweat function, levels of glucose, triglycerides, total cholesterol, HDL-cholesterol, LDL-cholesterol were determined, as the parameters of the metabolic syndrome. Univariate and multivariate analyzes were used to determine the relationship between these parameters and the neuropathy score determined by the sudoscan. Results: Of the 244 patients, aged 32 to 88 years, the average conductance of hands was $59.0 \pm 0.8 \mu \mathrm{S}$ and that of the feet $70.5 \pm 11.8 \mu \mathrm{S}$. The neuropathy score obtained was $39.5 \pm 16.2 \%$, with no significant difference between men and women $(\mathrm{p}=$ 0.289). By univariate analysis, age $(\mathrm{p}=0.0355)$, blood glucose $(\mathrm{p}=0.015)$, waist circumference $(\mathrm{p}=0.0006)$, the glycated hemoglobin $(\mathrm{p}=0.0378)$, the duration of diabetes $(\mathrm{p}=0.0006)$, systolic blood pressure $(\mathrm{p}=0.0378)$ and size $(\mathrm{p}=0.0062)$ were associated with neuropathy score. In multivariate analysis, there was an association between neuropathy score and the body mass index, weight, duration of diabetes, glycated hemoglobin and glycemia.

Conclusion: The conductance of the feet and hands as well as neuropathy score obtained by the sudoscan are associated with other parameters of the metabolic syndrome. The sudoscan can therefore provide information regarding to the glycemic control of diabetics.
\end{abstract}

Keywords: Type 2 diabetes, Sudoscan, Conductance, Metabolic syndrome, Neuropathy.

\section{Introduction}

Sub-Saharan Africa abounds in a disproportionate number of infectious diseases, but also no transmissible diseases such as diabetes. ${ }^{1}$ In 2011, the International Diabetes Federation estimated that 366 million people suffer from diabetes and another 280 million were at risk of developing this disease. This institution also provides that, this will increase from 280 to 552 million by $2030 .^{2}$ The World Health Organization considers diabetes as a pandemic whose prevalence increases considerably, including in Africa. ${ }^{3}$ This pathology is often associated with the metabolic syndrome, which is an association of several abnormalities whose definition differs according to the organisms. ${ }^{4}$ Diabetes and metabolic syndrome are linked to metabolic disorders including carbohydrate, lipid and protein with an imbalance between the contributions and energy expenditure. This imbalance leads to degenerative complications whose most affected organs are eyes, kidneys, nervous and vascular systems. ${ }^{5}$ Nowadays, this pathology, known as one of the fastest-growing chronic diseases, is well defined and easy to diagnose. However, the severity of type 2 diabetes is mainly based on its but more or less long term complications. Note the case of neuropathy is one of the complications that can lead to other ailments, such as diabetic foot ulcer or diabetic hand or dysfunction of the sweat glands. ${ }^{6}$ In fact, diabetes affects the small fibers of the peripheral nervous system which control the sweat glands, causing an ionic imbalance in the sweat channels of the sweat glands. ${ }^{7}$ The determination of the variation of chloride ions from the sweat may help to predict the onset of diabetes complications. ${ }^{8}$ Sudoscan's interest in screening for type 2 diabetes has been demonstrated by a previous study. ${ }^{9}$ This study aims to find relationships between sweat function assessed using the sudoscan and parameters of the metabolic syndrome in patients with diabetes type 2 in Libreville, Gabon.

\section{Material and Methods}

Ethical Consideration: This study was reviewed and approved by National Committee on Ethics for Research (CNER) of Gabon. Participants were informed that participation was completely voluntary, and written consent was obtained from each participant before being subjected to the questionnaire and after discussing the objective with the participants. All information collected was processed in strict compliance with anonymity and confidentiality of all patients. 
Area and Study Population: This study was conducted in the Department of Endocrinology of the University Hospital of Libreville (CHUL) and analysis of biological parameters were carried out in the laboratory of biochemistry at the University of Health Sciences in Libreville. This cross-sectional and descriptive study took place during the period from July to December 2016. The diabetes type 2 patients were involved and came for consultation in the Endocrinology Service of the CHUL. Only fasting people were included in this study.

Patients with acute or chronic infections, hospitalized patients and those who did not agree to participate in the study were not included. Enrolled patients were examined and the following parameters were identified: blood pressure, weight, height, abdominal perimeter, body mass index (BMI), capillary glycemia, and glycated hemoglobin determination from a capillary blood sample. The capillary blood is obtained from a drop of blood from the fingertip and applied to a specific strip. The result is given by an Accu-Check ${ }^{\circledR}$ player. In contrast, glycated hemoglobin is measured by using a SIEMENS DCA ${ }^{\mathrm{TM}}$ Systems reader.

Peripheral Neuropathy Research by Sudoscan: The sudoscan is a new-patented device, developed by the company $<<$ Impeto Medical Paris, France $>$. This is a non-invasive tool built for rapid assessment, quantitative and precise function of the sweat glands. It measures the conductivity by chloride ions from the sweat that use reverse iontophoresis. ${ }^{7}$ The method is based on the measurement of skin conductance, resulting from an electrochemical reaction, expressed in micro-siemens $(\mu \mathrm{S})$ between the chloride ions of sweat and the electrodes of the device in contact with the palm hands and the soles of the feet. The risk of developing peripheral neuropathy is provided from this conductance and additional biometric data. ${ }^{10}$

\section{Data Analysis}

Data were analyzed with Statistica logician. Quantitative data were expressed as means with standard deviation, while qualitative data were expressed as a percentage. The Mann Whitney Wilcoxon test was used to study the relationships between quantitative and qualitative parameters. The differences were significant for $\mathrm{p}$ values $<0.05$. Spearman's correlation coefficient was used to study the correlations between the quantitative variables and the neuropathy score. Multivariate analysis were used to visualize relationships between quantitative variables and neuropathy score.

\section{Results}

A total of 244 patients including 156 women, or $63.9 \%$. The minimum and maximum age were 32 to 88 years, then an average of $57 \pm 12.6$ years, with a sex ratio of 1.77 (Table 1).

Table 1: Biochemical and physical parameters

\begin{tabular}{|l|c|}
\hline \multicolumn{1}{|c|}{ Parameters } & Mean \pm Standard deviation \\
\hline Hand Conductance $(\mu \mathrm{S})$ & $59 \pm 0.8$ \\
\hline Feet Conductance $(\mu \mathrm{S})$ & $70.5 \pm 11.8$ \\
\hline Neuropathy score $(\%)$ & $39.5 \pm 16.2$ \\
\hline Weight $(\mathrm{Kg})$ & $76.4 \pm 2.0$ \\
\hline Size $(\mathrm{m})$ & $1.6 \pm 0.1$ \\
\hline Body mass index $(\mathrm{kg} / \mathrm{m} 2)$ & $28.8 \pm 5.5$ \\
\hline Waist $(\mathrm{cm})$ & $98.0 \pm 1.1$ \\
\hline Glycated hemoglobin $(\%)$ & $7.5 \pm 2.03$ \\
\hline Total cholesterol $(\mathrm{mmol} / \mathrm{L})$ & $4.2 \pm 1.0$ \\
\hline Cholesterol HDL $(\mathrm{mmol} / \mathrm{l})$ & $1.7 \pm 0.5$ \\
\hline Cholesterol LDL $(\mathrm{mmol} / \mathrm{L})$ & $2.0 \pm 0.8$ \\
\hline Triglyceride $(\mathrm{mmol} / \mathrm{L})$ & $1.1 \pm 0.4$ \\
\hline Capillary Glycemia $(\mathrm{mmol} / \mathrm{l})$ & $8.5 \pm 4.3$ \\
\hline Plasmatic Glycemia $(\mathrm{mmol} / \mathrm{l})$ & $8.4 \pm 3.3$ \\
\hline Duration of diabetes $($ years $)$ & $6.6 \pm 5.3$ \\
\hline Diastolic blood pressure $(\mathrm{mmHg})$ & $63.5 \pm 26.7$ \\
\hline Systolic blood pressure $(\mathrm{mmHg})$ & $137 \pm 20.8$ \\
\hline
\end{tabular}

BMI averaged $28.8 \pm 5.5 \mathrm{~kg} / \mathrm{m}^{2}$ corresponding to a waist circumference of $98.0 \pm 1.1 \mathrm{~cm}$. In terms of hand conductance the mean was $59 \pm 08 \mu \mathrm{S}$ versus to $70.16 \pm 11.8$ of that of the feet, with a neuropathy score of $39.5 \pm 16.2 \%$. The systolic blood pressure was higher than the diastolic with an average of $137 \pm 20.8$ versus $63.5 \pm 26.7 \mathrm{mmHg}$. Total cholesterol and LDL cholesterol were high at $4.2 \pm 1.0$ and $2 \pm 0.8 \mathrm{mmol} / \mathrm{L}$, respectively, with LDL cholesterol and low triglycerides at $1.7 \pm 0.51,1 \pm 0.4 \mathrm{mmol} / \mathrm{L}$, respectively. Neither conductance nor neuropathy scores were different between men and women (Table 2). 
Table 2: Distribution of conductances by gender

\begin{tabular}{|l|c|c|c|}
\hline Parameters & Women & Men & $\boldsymbol{p}$ \\
\hline Feet conductance $(\mu \mathrm{S})$ & $69.5 \pm 11.9$ & $73.9 \pm 8.9$ & 0.151 \\
\hline Hand Conductance $(\mu \mathrm{S})$ & $58.2 \pm 15.7$ & $61.8 \pm 19.6$ & 0.441 \\
\hline Neuropathy score & $41.2 \pm 13.7$ & $33.6 \pm 19.8$ & 0.289 \\
\hline
\end{tabular}

The study of quantitative parameters correlated with the neuropathy score (Table 3 ) revealed the role of the subject's age (Rho $=0.4272, \mathrm{p}=0.0006)$, waist circumference $(\mathrm{Rho}=0.285 ; \mathrm{p}=0.0003)$ and patient size $(\mathrm{Rho}=-0.3463, \mathrm{p}=0.0062)$.

According to the biochemical markers, only plasmatic Glycemia (Rho $=0.3100 ; \mathrm{p}=0.015$ ); glycated hemoglobin $($ Rho $=0.4300, p=0.0378)$ were positively correlated with neuropathy score.

Neuropathy is positively correlated with waist circumference, and therefore it is strongly linked to obesity. This result was confirmed by multivariate analysis (Fig. 1).

In this multivariate analysis, the percentage variance was $67 \%$. The results showed that the percentage of neuropathy was positively associated with the glycated hemoglobin percentage on the second axis and the plasmatic Glycemia. On the other hand, on the first factorial axis, this neuropathy was positively associated with the parameters defining obesity (BMI, Weight) and negatively with the conductances.
Table 3: Results of spearman correlations with neuropathic score

\begin{tabular}{|l|c|c|}
\hline \multicolumn{1}{|c|}{ Studied Parameters } & Rho & p \\
\hline Age & 0.4272 & 0.0006 \\
\hline BMI & 0.2698 & 0.0355 \\
\hline Plasma Glycemia & 0.3100 & 0.015 \\
\hline Waist size & 0.2857 & 0.0006 \\
\hline HBA1C & 0.4300 & 0.0378 \\
\hline Duration of diabetes & 0.2808 & 0.0003 \\
\hline Feet Conductance & -0.4452 & 0.5283 \\
\hline Hand conductance & -0.0823 & 0.0096 \\
\hline Systolic pressure & 0.3291 & 0.0378 \\
\hline LDL & 0.0139 & 0.9172 \\
\hline TG & 0.0773 & 0.5538 \\
\hline HDL & 0.2066 & 0.1102 \\
\hline TC & -0.1023 & 0.4328 \\
\hline Size & -0.3463 & 0.0062 \\
\hline Weight & 0.1164 & 0.3718 \\
\hline
\end{tabular}

Legend: TC (total cholesterol); LDL (Low-density lipoprotein cholesterol); HDL (high-density lipoprotein cholesterol); TG (triglycerides); HBA1C (glycated hemoglobin); BMI (body mass index).

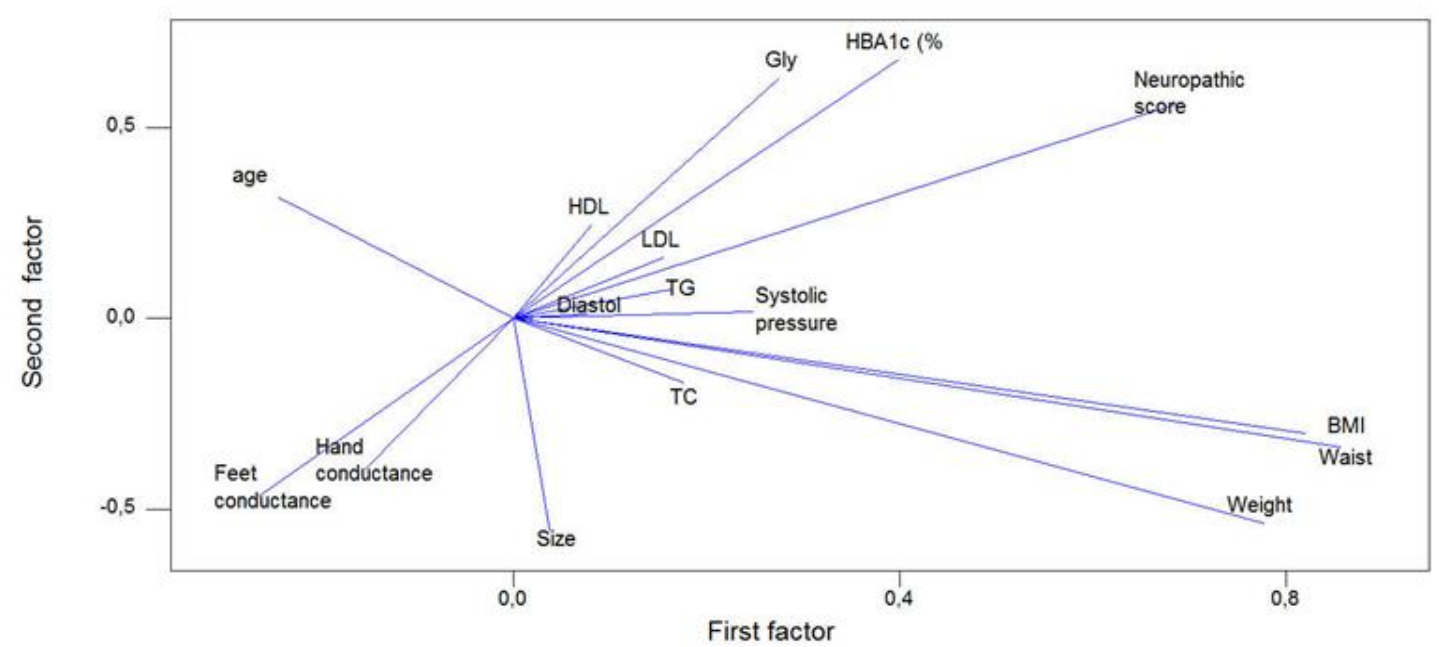

Fig. 1: Results of the analysis. DBP (diastolic blood pressure); BMI (body mass index); TG (triglycerides); Gly (Glycemia) HbA1C (glycated hemoglobin); HDL (High density lipoprotein cholesterol); LDL (Low density lipoprotein cholesterol).

\section{Discussion}

The purpose of this study was to make a correlation between the parameters defining metabolic syndrome and the sweat function in type 2 diabetic patients. Except lipid parameters, all other parameters involved in the definitions of the metabolic syndrome are linked to neuropathy score and were determined by sudoscan.
The sudoscan is a device for measuring the cutaneous electrochemical conductivity, developed by IMPETO de France. The sudomotor function is evaluated using reverse iontophoresis and chronoamperometry. It allows the electrochemical measurement of cutaneous conductance of the palms of the hands and soles of the feet when a low voltage current is applied between the electrodes..$^{11}$ It has been 
shown that the sudoscan could be used as an early diagnostic tool of distal symmetrical neuropathy. ${ }^{12}$ Now this polyneuropathy is one of the most common complications and debilitating diabetes.

Relations between neuropathy score with one hand, the blood glucose, and secondly with glycated hemoglobin have been established. This relationship is based on the fact that the chronic hyperglycemia of diabetes is responsible for the alteration of the microvessels, which is itself responsible for the sensorimotor and symmetrical polyneuropathy of the diabetic subject. ${ }^{13}$ These results confirm those of Bouenizabila et al. ${ }^{9}$

The other clinical parameters of the metabolic syndrome are also related not only to the neuropathy score, but also to the cutaneous electrochemical conductance of the hands and feet. This result confirms that of Zhu et al who worked in a Chinese population. ${ }^{14}$ These authors demonstrated that the effect intensity of autonomic dysfunction determined by the sudoscan, was proportional to the number of components of the metabolic syndrome.

On the other hand, there appears to be no relationship between the electrochemical conductance of the hands or feet and the lipid components of the metabolic syndrome. Two hypotheses may justify this observation. The first is based on the relatively low proportion of patients with dyslipidemia in this study. Indeed, only $10.2 \%$ of the study population had hypertriglyceridemia, and among these subjects, none had a decreased concentration of HDL cholesterol. In fact, this observation corresponds to the low rate of dyslipidemia classically found in type 2 diabetics. Other authors have demonstrated that triglycerides, for example, are a bad marker of the metabolic syndrome in African populations. ${ }^{15,16}$ The second hypothesis may explain the lack of relationship between the concentration of lipids and neuropathy comes from the fact that the initial disturbance of lipids due to chronic hyperglycemia rather involve oxidation of lipoproteins, including LDL. ${ }^{17}$

This study highlights a relationship between diabetic neuropathy and patient weight, waist circumference, and body mass index. These parameters are indeed elements commonly found in type 2 diabetes, although their frequency is lower in African than European populations. ${ }^{18}$

The relationship between patient age and neuropathy is relatively important in this study, which was conducted from a population of diabetics. However, Vinik et al ${ }^{19}$ working with a non-diabetic population, found only a weak relationship between electrochemical conductance and patient age. Type 2 diabetes would therefore accentuate this relationship and thus peripheral autonomic nerve damage.

The relationship between neuropathy score, duration of diabetes and glycated hemoglobin is fundamental in monitoring the diabetic patient. Indeed, it suggests that the control of diabetes and its complications including renal can be performed using the sudoscan. This device would therefore be useful for not only the early diagnosis of diabetes, but also for monitoring diabetes and its complications, as suggested by Mao et al. ${ }^{20}$

This study displayed some limits. The first one was the nature of the study. This was not a case-control study, even if 244 subjects composed the sample. The second aspect concerned the cost of this device $(20,000$ USD \$). Certainly the used of sudoscan will be restricted for all hospitals and laboratories from a resource-poor like ours.

\section{References}

1. Claude J. Le diabète sucré en Afrique: enjeu de santé publique. Bulletin de l'Académie Nationale de Médecine 2011;195(6):1239-53.

2. International Diabetes Federation 2011. Atlas du diabète $5^{\mathrm{e}}$ édition, Bruxelles.

3. Hall V, Thomsen RW, Henriksen O. Diabetes in Subsaharan Africa 1999-2011: epidemiology and public health implications. A systematic review. BMC Public Health. 2011;11:564.

4. Marksimovic MZ, Vlajinac HD, Radak DJ. Prevalence of the metabolic syndrome in patients with carotid disease according to NHLBI/AHA and IDF criteria: a crosssectional study. BMC Cardiovasc Disord. 2012;12:2.

5. Lu B, Hu J, Wen J. Determination of peripheral neuropathy prevalence and associated factors in Chinese subjects with diabetes and pre-diabetes - Shang Hai Diabetic neu Ropathy Epidemiology and Molecular Genetics Study (SH-DREAMS). PLoS One. 2013;8(4):e61053.

6. Ho YN, Lee KO, Jeong J. The role of insulin resistance in diabetic neuropathy in Koreans with type 2 diabetes mellitus: A 6-year follow-up study. Yonsei Med J. 2014;55(3):700-8.

7. Khalfallah K, Ayoub H, Calvet JH. Noinvasive galvanic skin sensor for early diagnostic of sudomotor dysfunction: application to diabetes. IEEES ens $J$. 2012;12:456-63.

8. Bauduceau B, Mayaudon H, Garcia C. O68 Le SUDOSCAN: un nouvel outil pour la détection précoce de la neuropathie autonome cardiaque: comparaison aux méthodes classiques d'exploration. Diabetes and Metabolism. 2011;37(1):A17.

9. Bouenizabila E, Kakou C, Bauduceau B. Dépistage du diabète de type 2 et de ses complications en Afrique subSaharienne : la place potentielle du sudoscan. Médecine des Maladies Métaboliques. 2015;9(2):165-70.

10. Yajnik CS, Kantikar VV, Pande AJ. Quick and simple evaluation of sudomotor function for screening of diabetic neuropathy. ISRN Endocrinol. 2012;2012:103714.

11. Schwartz PE, Brunswick P, Calvet PH. EZSCAN a new technology to detect diabetes risk. Br J Diabetes Vasc Dis. 2011;11:204-9.

12. Smith AG, Lessard M, Reyna S. The diagnostic utility of Sudoscan for distal symmetric peripheral neurpathy. $J$ Diabetes Complications. 2014;28(4):511-16.

13. Tesfaye S, Boulton AJM, Dyck PJ. On behalf The Toronto Diabetic neuropathy Expert group. Diabetic neuropathies: update on definitions, diagnostic criteria, estimation of severity and treatments. Diabetes Care. 2010;33:2285-93. 
14. Zhu L, Zhao X, Zeng P. Study on autonomic dysfunction and metabolic syndrome in Chinese patients. J Diabetes Investig. 2016; doi:10.1111/Jdi.12524.

15. Yu SS, Ramsey NL, Castillo DC. Triglyceride-based screening test fail. Co-recognize cardiometabolic disease in African immigrant and African-American men. Met Syndr Relat Discord. 2013;11(1):15-20.

16. Knight MG, Goedelecke JH, Ricks M. The TG/HDL-C ratio does not predict insulin resistance in overweight women of African descend: a study of South African: A study of South African and west African women. Ethn Dis. 2011;21(4):490-4.

17. Macut D, Danijanovic S, Panidis D. Oxidised low-density lipoprotein concentration - early marker of an altered lipid metabolism in young women with PCOS. Eur J Endocrinol. 2006;155(1):131-6.

18. Ovono Abessolo F, Bekale S, Fernandez J. Cardiovascular risk factor in type 2 diabetic patients in Libreville, Gabon". African J Diabetes Medicine. 2011;19(2):12-4
19. Vinik AI, Smith AG, Singleton JR. Normative Values for electrochemical Skin conductances and Impact of ethnicity on quantitative Assessment of sudomotor function. Diabetes Technol Ther. 2016;8(6):391-8. doi: 10.1089/dia.2015.0396.

20. Mao F, Liu S, Qiao X. Sudoscan, an effective tool for screening chronic kidney disease in patient with type 2 diabetes. Exp Ther Med. 2017;14(2):1343-50.

How to cite this article: Ovono Abessolo F, Mbang Bengone AS, Ndong Atomo GR, Batou AS, Ngou Milama E. Relationship between sweat function determined by the sudoscan and parameters of the metabolic syndrome in people with type 2 diabetes. Int $\mathrm{J}$ Clin Biochem Res. 2018;5(4):560-564. 